\title{
Editorial
}

\section{Martina Zaninotto and Mario Plebani* \\ Chest pain management and biomarkers: the lack of trust in cardiac troponins measurement}

https://doi.org/10.1515/dx-2020-0156

The recently published guideline from the European Society of Cardiology confirms yet again the role of cardiac troponins (measured with high-sensitivity methods) as the biochemical gold standard for the diagnosis of acute coronary syndrome, and recommends the discontinuation of CK, CK-MB, H-FABP and copeptin measurements from the routine diagnostic biochemical panel [1]. Since the year 2000, when the redefinition of myocardial infarction was published [2], various recommendations have been issued by both clinical and laboratory scientific societies and several original papers have also been published [3, 4]. All suggest eliminating the use of CK-MB and other biomarkers for diagnostic purposes collecting further evidence over time.

The paper by Ilardi et al. [5] appearing in the current issue of the Journal, reports interesting data from a survey conducted in medical wards in Italy, in the Campania region in particular; the aim was to assess the biomarkers routinely used in the management of patients with chest pain. The results demonstrate that $26.8 \%$ of clinics responding to questionnaire preferred the simultaneous measurement of cardiac troponins (I or T) and CK-MB, $32.9 \%$ use the additional myoglobin assay, and only $30.5 \%$ use troponin measurement alone, considering it the unique, most appropriate available test. Furthermore, 9.8\% used a biochemical panel that includes "cTns, total CK, CK-MB, myoglobin and LDH”. However, the paper does not provide information on the adoption of cardiac troponins serial testing for detecting the origin of chest pain as recommended by the guidelines.

Similar surveys, promoted by both cardiology and laboratory medicine scientific societies, were conducted in Italy before the publication of "Redefinition of myocardial infarction" [1], one in 1999 by ANMCO [6] and another in

\footnotetext{
*Corresponding author: Mario Plebani, Department of Laboratory Medicine, University Hospital, Padova, Italy, E-mail: mario.plebani@ unipd.it. https://orcid.org/0000-0002-0270-1711

Martina Zaninotto, Department of Laboratory Medicine, University Hospital, Padova, Italy
}

2005, by SiBioC [7]. The end-points of these surveys, carried out across the whole set of Italian Coronary Care Units [6] and Laboratory Medicine departments [7] were to evaluate: (1) the approach of clinicians in detecting myocardial necrosis using available biomarkers; (2) the clinical and laboratory management of the total quality process with the adoption of new biomarker assays, in particular cardiac troponins; (3) the consensual strategy of clinical and laboratory professionals in using the new biochemical panel for the management of patients with chest pain. The results of both surveys demonstrated that cardiac troponins measurement was widely used (by $70-100 \%$ of responders), albeit in association with a panel of outdated biomarkers (CK, AST and LDH by $66-94 \%$ of the responders), with CK-MB by $71-100 \%$ and myoglobin by $44 \%$ of the responders, the most commonly used panel being "cTns+CK+CK-MB+LDH+AST" (44\%). Furthermore, no differences in the use of biomarkers were observed in relation to the clinical or laboratory setting, except for the case of cardiac troponins, which were more frequently used in a more complex clinical context.

Twenty years on, the data reported in the present survey demonstrate that clinicians working in medical wards continue to suffer from a lack of confidence in cardiac troponins measurement: $70 \%$ currently use a combination of outdated and no longer recommended biomarkers such as myoglobin, LDH and CK, the preferred combination being cardiac troponins and CK-MB. In the paper, furthermore, no information is given on the behavior pattern of requesting, whether in association (series) or in parallel.

It is well known that CK-MB has a significantly lower diagnostic specificity and sensitivity than cardiac troponins: the 99th percentile value of cardiac troponins in healthy subjects corresponds to the amount of the biomarker in about 20-40 mg of myocardial tissue, a volume that is too low to be detected by other currently available laboratory tests, and even by non-invasive cardiac imaging [8]. Therefore, the potential clinical advantage of a highly sensitive and cardio-specific diagnostic test, such as cardiac troponins, appears to be completely lost if it is combined with CK-MB in the biochemical predictive strategy, with a 
consequent failure to identify patients with myocardial injury at risk of serious cardiovascular complications [9].

Importantly, the survey was carried out in general Departments of Medicine of medium-sized hospitals, where the small number of beds and the case-mix call for a more widespread commitment to diagnostic and therapeutic appropriateness. On the other hand, in the specific clinical setting considered, laboratory professionals demonstrated a weak strategy and commitment to assuring advice to clinicians on more appropriate test requesting based on biochemical characteristics and analytical performances [10]. Therefore, the most relevant finding made seems to be the poor diffusion of available scientific evidence and guidelines focusing on the clinical value of cardiac troponins measurement in patients with chest pain: the high positive predictive value of this approach allows the rapid diagnosis of acute cardiac injury with an improved clinical outcome, while the significant negative predictive value ensures cardiac damage can be safely and rapidly ruled out, thus enabling the accurate classification of patients, and the optimization of clinical and organizational pathways [11].

The above advantages are lost on adding CK-MB measurement, the results of which nullify the specific and sensitive clinical information provided by cardiac troponins. Today, a multidisciplinary, shared strategy [12] should be used to address the urgent need to reappraise biochemical pathways for chest pain management.

Research funding: None declared.

Author contributions: All authors have accepted responsibility for the entire content of this manuscript and approved its submission.

Competing interests: Authors state no conflict of interest.

\section{References}

1. Collet JP, Thiele H, Barbato E, Barthelemy O, Bauersachs J, Bhatt DL, et al. 2020 ESC guidelines for the management of acute coronary syndromes in patients presenting without persistent ST-segment elevation. Eur Heart J 2020. https://doi.org/10.1093/eurheartj/ ehaa575.
2. The Joint European Society of Cardiology/American College of Cardiology Committee. Myocardial infarction redefined - a consensus document of the Joint European Society of Cardiology/ American College of Cardiology committee for the redefinition of myocardial infarction. J Am Coll Cardiol 2000;36:959-69.

3. Wu AHB, Christenson RH, Greene DN, Jaffe AS, Kavsak PA, Ordonez-Llanos JO, et al. Clinical laboratory practice recommendations for the use of cardiac troponin in acute coronary syndrome: expert opinion from the Academy of the American Association for Clinical Chemistry and Task Force on clinical applications of cardiac bio-markers of the International Federation of clinical Chemistry and Laboratory Medicine. Clin Chem 2018;64: 645-55.

4. Mueller C, Giannitsis E, Mockel M, Huber K, Mair J, Plebani M, et al. Rapid rule-out of acute myocardial infarction: novel biomarker-based strategy. Eur Heart J Acute Cardiovasc Care 2017;6:218-22.

5. Ilardi A, Caruso G, Ilardi C, D'Avino N. Chest pain management: use of troponins in Internal Medicine wards. Diagnosis (Berl) 2021;8: 388-91.

6. Ottani F, Galvani M, Dolci A, Plebani M, Tubaro M, Zaninotto M, et al. I marcatori di danno miocardico nella diagnosi di infarto miocardico acuto: la realtà italiana nell'anno 2000. Ital Heart J 2002;3:933-42.

7. Galli GA, Gambetta C, Caputo M, Cappelletti P. Survey on the use of cardiac markers in Italy. IJLaM 2005;1:46-55.

8. Marjot J, Kaier TE, Martin DE, Reji SS, Copeland N, Iqbal M, et al. Quantifying the release of biomarkers of myocardial necrosis from cardiac myocytes and intact myocardium. Clin Chem 2017; 63:990-6.

9. Shah ASV, Anand A, Strachan FE, Ferry AV, Lee KK, Chapman AR, et al. High-sensitivity troponin in the evaluation of patients with suspected acute coronary syndrome: a stepped-wedge, clusterrandomised controlled trial. The lancet.com. https://doi.org/10. 1016/50140-6736(18)31923-8.

10. Vittorini S, Clerico A. Cardiovascular biomarkers: increasing impact of laboratory medicine in cardiology practice. Clin Chem Lab Med 2008;46:748-63.

11. Ambavane A, Lindahl B, Giannitsis E, Roiz J, Mendivil J, Frankenstein $L$, et al. Economic evaluation of the one-hour ruleout and rule-in algorithm for acute myocardial infarction using the high-sensitivity cardiac troponin T assay in the emergency department. PLoS One 2017;12:e0187662.

12. Julicher P, Greenslade JH, Parsonage WA, Cullen L. The organisational value of diagnostic strategies using highsensitivity troponin for patients with possible acute coronary syndromes: a trial-based cost-effectiveness analysis. BMJ Open 2017;7:e013653. 